\title{
Camera Sensor Based Human Gait Analysis for Ubiquitous Healthcare Monitoring System
}

\author{
Young-Sook Lee ${ }^{1}$, Wan-Young Chung ${ }^{2}$ \\ ${ }^{1}$ Electronic Information Communication Research Center, Pukyong National University, 608-737, \\ South Korea, \\ ${ }^{2}$ Department of Electronic Engineering, Pukyong National University, 608-737, South Korea \\ wychung@pknu.ac.kr
}

\begin{abstract}
:
A novel camera sensor based algorithm for automated human gait recognition using computer vision technology is presented. We develop a real-time camera sensor based ubiquitous healthcare monitoring system for human gait recognition that employs gait feature information such as the stride length variations, gait cycle time and the angle variations from the detected subject's center point and to end point of feet. Feature extraction in videos is an essential topic in object detection and recognition, as it helps the algorithm to improve the system performance for recognition rates. Experiments performed with video streams show efficient and reliable gait analysis with our approach.
\end{abstract}

Key words: Human Gait Recognition, Camera Sensor, Ubiquitous Healthcare Monitoring System, Feature Extraction, Motion Analysis, and Abnormal Behavior.

\section{Introduction}

In recent years, with the development of widespread availability of digital cameras and techniques of intelligent video analysis, camerabased human gait analysis has been one of the active as well as challenging research issues. Until now, many pervious techniques for gait recognition have addressed in the literature. As a biometric, human gait is defined as a means of identifying individuals by the way they walk [1-2]. Using gait has many advantages over other biometrics, such as fingerprints, iris, and face recognition, most notably because it is noninvasive and available at low resolution.

Many related works have been presented in the literature for distinguishing, predicting, predicting, and monitoring moving human object of interests. Diverse visual cues such as movements typically characterizing the alertness of patients or people are extracted in real time. Camera sensor based schemes have been extensively proposed because of its accuracy, robustness, and non-intrusiveness.

Our proposed system to the camera based human gait analysis is divided into background modeling, foreground detection, and gait feature extraction, and human gait analysis as shown in Fig. 1. The objective of our algorithm is to extract gait features for analyzing human gaits. To realize accurate and robust detection for a moving human body as a foreground, it is effective to utilize background subtraction based adaptive background modeling. Moment analysis is used to extract gait features such as the stride length variations and the angle variations from the detected subject's center point and to end point of feet.

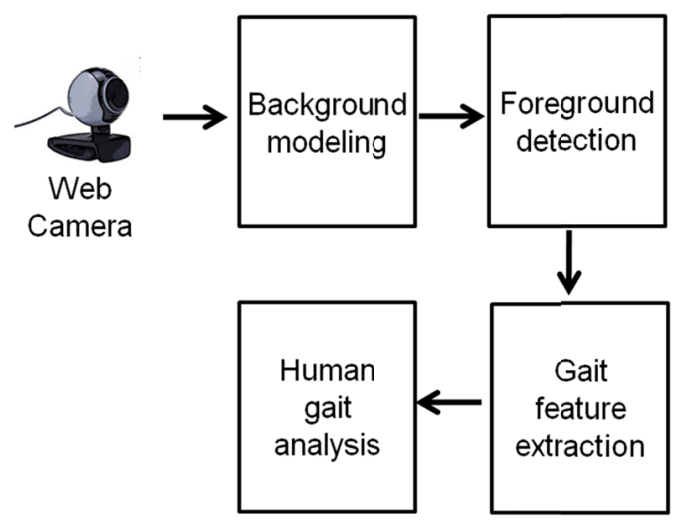

Fig. 1. Block diagram of proposed human gait analysis system.

\section{Approach}

Foreground detection is an important process for a gait recognition system because it will influence the final detection results. Background subtraction is the common technique for motion segmentation in static scienes [3]. There are many existing methods of background subtraction for foreground extraction such as non-parametric model, a single Gaussian model, mixture Gaussian model, and so on [3]. But our 
method utilizes averaging background method that is computationally faster than others. In order to extract the silhouettes of the moving subjects from the background in the video sequence, a simple and fast background subtraction technique using the average value is adopted to construct the background model from a small portion of video sequence including moving objects. It attempt to detect moving regions by subtracting a current image pixel-by-pixel from a background image that is created by averaging images over time in an initialized period. At time $t$, the background is calculated as follows:

$$
B_{t}(x, y)=\alpha I_{t-1}(x, y)+(1-\alpha) B_{t-1}(x, y)
$$

where $B_{t}(x, y)$ represents the updated pixel value of background at pixel position $(x, y)$ at time $t$. $I_{t}(x, y)$ stands for current image pixel value at time $\mathrm{t}$, and $\alpha$ is a weighting parameter between 0 and 1 .

After the background is generated, the foreground pixel can be extracted by the following equation:

$$
F_{t}(x, y)=\left\{\begin{array}{cc}
1 & \left|I_{t}(x, y)-B_{t}(x, y)\right|>T \\
0 & \text { otherwise }
\end{array}\right.
$$

where $T$ is a predefined threshold. If the difference image is greater than the threshold, then the pixel is labeled as foreground, while background pixels are modified by eq. (1).

To reduce the effects of noise and enhance the detected regions, some morphological post processing operations such as erosion, dilation are performed after creating foreground extraction. Connected component analysis is commonly used to refer to the process of detecting connected objects in an image. This task is to segment an object from background on the image. A typical connected component analysis is applied to the binary image obtained from eq. (2).

Feature extraction is one of the major important issues in many visual applications. We consider gait features to analyze human gaits in our algorithm. The features include the stride length variations, gait cycle time, a binary silhouette and the angle variations and the angle variations from the detected subject's center point and to end point of feet. To compute the angle, object tracking and recognition algorithm in video is to search for the centroid and an extent of bounding box. The bounding box is an expression of the maximum extents of a 2- dimensional object within its 2-D $(x, y)$ coordinate system.

A lot of useful information about the binary object can be gained from the moments of the object. In order to find the extent of and centroid of a segmented foreground region, area of the segmented object is obtained by the moments. For every image sequences, an approximate bounding box is fitted to the motion region obtained by foreground segmentation. The fitting blob can be computed by using moments [4]. For an image $I_{t}(x, y)$, the moments are given by: $M_{p g}=\sum_{(x, y) \in \text { Pixels }} x^{p} y^{q} I(x, y)$ where $\mathrm{p}, \mathrm{q}=0,1,2 \ldots .$. We can obtain the centroid $(\bar{x}, \bar{y})$ of the bounding box by: $\bar{x}=M_{10} / M_{00}$ and $\bar{y}=M_{01} / M_{00}$.

We develop a real-time camera sensor based ubiquitous healthcare monitoring system for human gait recognition that employs gait feature information such as the stride length variations and the angle variations from the detected subject's center point and to end point of feet. The angle is one of features which analyze human gaits as shown in Fig. 2. The angle variation in normal behavior is greater than that of abnormal behavior in such as Parkinson's patient.

The angle $\theta$ can be calculated as

$$
\begin{aligned}
& \theta=\left(90^{\circ}-\alpha\right) \times 2 \\
& \alpha=\cos ^{-1}\left(\frac{b}{\sqrt{a^{2}+b^{2}}}\right) \\
& \text { 国 output }
\end{aligned}
$$

Fig. 2. The angle $\theta$ is one of features for gait analysis.

Gait is treated as a periodic activity within each gait cycle time. A single gait cycle can be regarded as the time between two identical events during the person walking and usually measured from heel strike to heel strike of one leg. Fig. 3 shows the result of gait cycle and stride length variation for normal and abnormal person walking. Compared with normal people, PD patients' walking speed is slower, stride 
length is shorter, duration of gait cycle is longer, and amplitude of range of movement of joints is decreased.

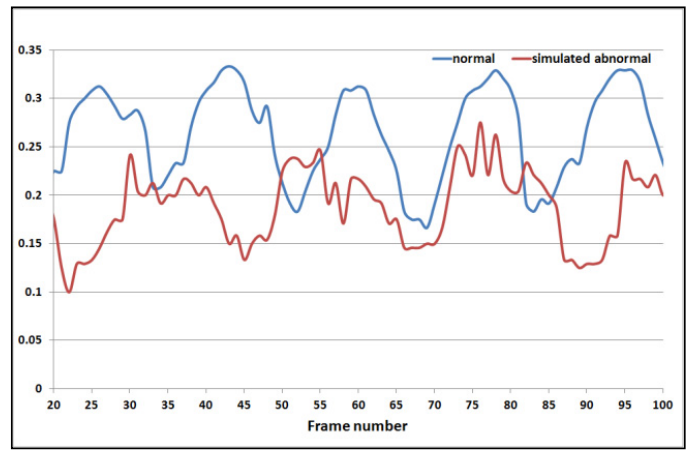

Fig. 3. Result of stride length variation for normal and simulated abnormal person walking.

Human tracking is to achieve more accurate moving objects in an image sequence. The predictive filter is used to predict the position of the bounding box for the person and match it with the closest a moving object as the foreground.

\section{Experimental results}

The feasibility of a novel camera sensor based gait analysis for the proposed method is tested using image sequences in real time. The test image sequences are taken by the camera sensor at a frame rate 15-25 frames per second with $320 \times 240$ pixel resolution. The database includes a total of 20 gait sequences that are 5 simulated abnormal and 15 normal walking videos.

Experimental tests have proven to be a reliable and robust analysis method for classifying the individual gait patterns. An efficient gait analysis system for ubiquitous healthcare monitoring human applications by extracting features information including the stride length variations, cycle time, and the angle variations from the detected subject's center point and to end point of feet is used. The objective of this approach is to detect good feature extraction for gait recognition in real-time. Our proposed gait recognition algorithm can be applied in a ubiquitous healthcare video monitoring system to distinguish the gait patterns of Parkinson's disease patients from normal people.

To recognize human by gait motion, a PCA algorithm is employed as a classifier, and a small number of gait features are utilized as input data [5]. We have mainly used stride length, the angle, gait cycle time, and binary silhouette. The features extracted from image sequences during a time periodic. Even though the recognition rate is $95 \%$, our algorithm is tested in a very small dataset including image sequences.

Fig. 4 shows some results of our proposed camera sensor based system for gait analysis. Detected and tracked person is marked with a rectangle on the output frame (Fig.4c).

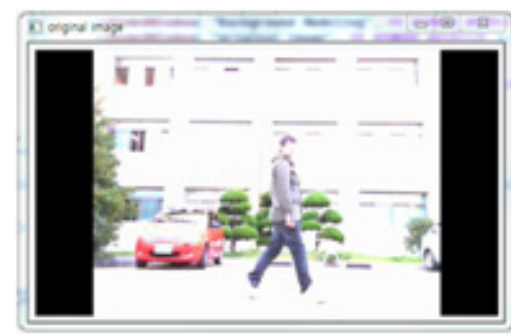

(a)

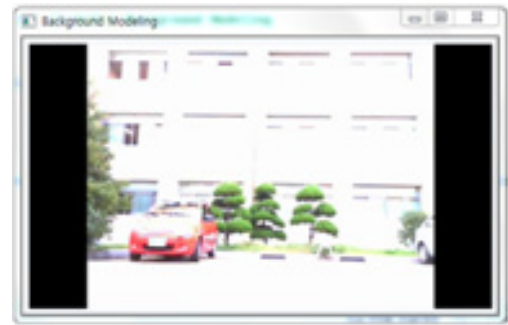

(b)

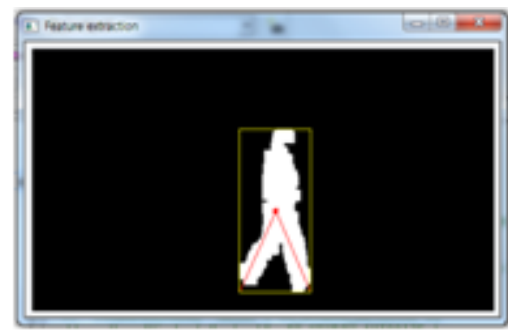

(c)

Fig. 4. Results of our proposed camera sensor based system for gait analysis (a) Original image (b) Background modeling, and (c) Extracted foreground image and gait features.

\section{Conclusion}

A novel camera sensor-based scheme is proposed to analyze human gait for ubiquitous healthcare monitoring system using gait features. Our proposed algorithm can be applied to discriminate the gait patterns of Parkinson's disease patients from normal people.

Feature extraction in videos is an essential topic in object detection and recognition, as it helps the algorithm to improve the system performance for recognition rates. The experimental results showed that our novel approach based on camera sensor was able to achieve a good detection success rate. In order to improve our method, we need to extend the analysis in future. 


\section{Acknowledgements}

This research was supported by Basic Science Research Program through the National Research Foundation of Korea (NRF) funded by the Ministry of Education, Science and Technology (2011-0005972).

\section{References}

[1] L. Wang, G. Y. Zhao, N. Rajpoot, M. Nixon, Special issue on new advances in video-based gait analysis and applications: challenges and solutions, IEEE Transactions on System, Man and Cybernetics-Part B (TSMC-B) 40(4), 982985(2010); doi: 10.1109/ TSMCB. 2010. 2046351

[2] J. Lu and Y. P. Tan, Gait based human age estimation, IEEE Transactions on System, Information Forensics and Security 5(4), 761-770 (2010); doi: 10.1109 /TIFS. 2010. 2069560.

[3] Background subtraction techniques: a review, IEEE International Conference on Systems Man and Cybernetics 4, 3099 - 3104(2004); doi: 10.1109/ICSMC. 2004. 1400815

[4] I. Pitas, Digital Image Processing Algorithms and Applications, New York, WILEY (2000).

[5] Vidal, R.; Yi Ma; S. Sastry, Generalized principal component analysis (GPCA), Proceedings. 2003 IEEE Computer Society Conference on Computer Vision and Pattern Recognition, I-621 - I-628(1), 2003; doi: 10.1109/CVPR.2003.1211411. 